\title{
Observation of Medium Scale Traveling lonospheric Disturbances (MSTIDs) in the low latitudes Brazilian sector using lonosonde data in comparison with optical measurements
}

\author{
'Danielle C. M. Amorim, ${ }^{1}$ Alexandre A. Pimenta
}

${ }^{1}$ National Institute for Space Research (INPE) - Av. dos Astronautas 1758, São José dos Campos, SP, Brazil danielle@laser.inpe.br, pimenta@laser.inpe.br

\begin{abstract}
In this paper we report observations of MSTIDs in the low latitudes Brazilian sector using ionograms obtained by a digisonde DGS256 and all-sky images of OI $630 \mathrm{~nm}$ airglow emission. Both instruments are installed at Cachoeira Paulista $\left(22.7^{\circ} \mathrm{S}, 45^{\circ} \mathrm{W}, 13^{\circ} \mathrm{S}\right.$ MLAT). Ionograms show occurrence of spread-F and a sharp rise of the ionospheric F-layer at the same time as dark band structures propagating northwestward are seen on all-sky images. Here, we show a study based on ionogram analysis in order to investigate occurrences of MSTIDs during solar maximum, since they are not observed on all-sky images during such period.
\end{abstract}

\section{Introduction}

MSTIDs have been observed in the low latitudes Brazilian sector using ionograms and all-sky images of OI $630 \mathrm{~nm}$ nightglow emission. On all-sky images they are seen as dark band structures which propagates from southeast to northwest with an average speed of 50-200 m/s at an altitude range of 220-300 km [1-2]. In a statistical analysis of all-sky images covering all phases of the solar cycle, [3] observed maximum occurrence of MSTIDs during solar minimum, but no occurrence during solar maximum. It was also observed maximum occurrence of MSTIDs during local winter and all cases detected occurred during geomagnetically calm nights.

Simultaneous airglow and ionosonde data show sharp risings of F-layer at the same time that dark band structures are recorded in the all-sky images over the zenith. The dark band pattern refers to depletions in the OI 630 $\mathrm{nm}$ emission due to vertical movements of the F-layer. Since the OI $630 \mathrm{~nm}$ emission intensity is strongly dependent on the electronic concentration, its tendency is to decrease or increase as the F-layer plasma moves upward or downward. Ionograms also show the occurrence of spread-F during MSTIDs events.

In this work we present a statistical study based on the analysis of ionograms for the period of solar maximum, which was made in order to investigate the possible occurrence of MSTIDs during this phase of the solar cycle. Our hypothesis of risings not so sharp of the F-layer could justify the non-observance of dark band structures in the all-sky images during solar maximum since it would not disturb the airglow layer.

\section{Data analysis}

Our research work is based on three types of analysis with data obtained by an all-sky imager and a digisonde DGS 256, both installed at Cachoeira Paulista $\left(22.7^{\circ} \mathrm{S}, 45^{\circ} \mathrm{W}, 13^{\circ} \mathrm{S}\right.$ MLAT). In the first one we analyze only all-sky images. The results obtained show some features common to all events. These features are the preferential alignment (southwest-northeast) and propagation (northwestward) directions of the dark bands and the occurrence during geomagnetically quiet nights $(\mathrm{Kp}<3)$.

The second analysis was done by comparing simultaneous data from both all-sky images and ionograms. The results show sharp rises of the F-layer and the occurrence of spread-F during MSTIDs events.

The third analysis was made using only ionograms in order to verify if the risings of the F-layer were not so sharp so that it could not disturb the emission. Such fact could justify the non-observance of dark bands during solar maximum. The last two analyses are treated in this work. 


\subsection{Comparisons between both airglow images and ionograms}

Figure 1a shows a plot of the temporal variation of ionospheric parameters h'F and $h m F 2$, and Figure $1 \mathrm{~b}$ shows a sequence of raw all-sky images in the OI $630 \mathrm{~nm}$ emission, both obtained on 23-24 August, 2006, for a geomagnetically quiet night $(\mathrm{Kp}<3)$. The digisonde observations registered abrupt increases in both the F-layer peak height (hmF2) and F-layer bottomside virtual height (h'F) on 23-24 August 2006, when the low intensity band passed over Cachoeira Paulista. From simultaneous analysis we can see that the dark band structure observed in the all-sky images corresponds to zones of depleted airglow due to the uplift of ionospheric F-layer.
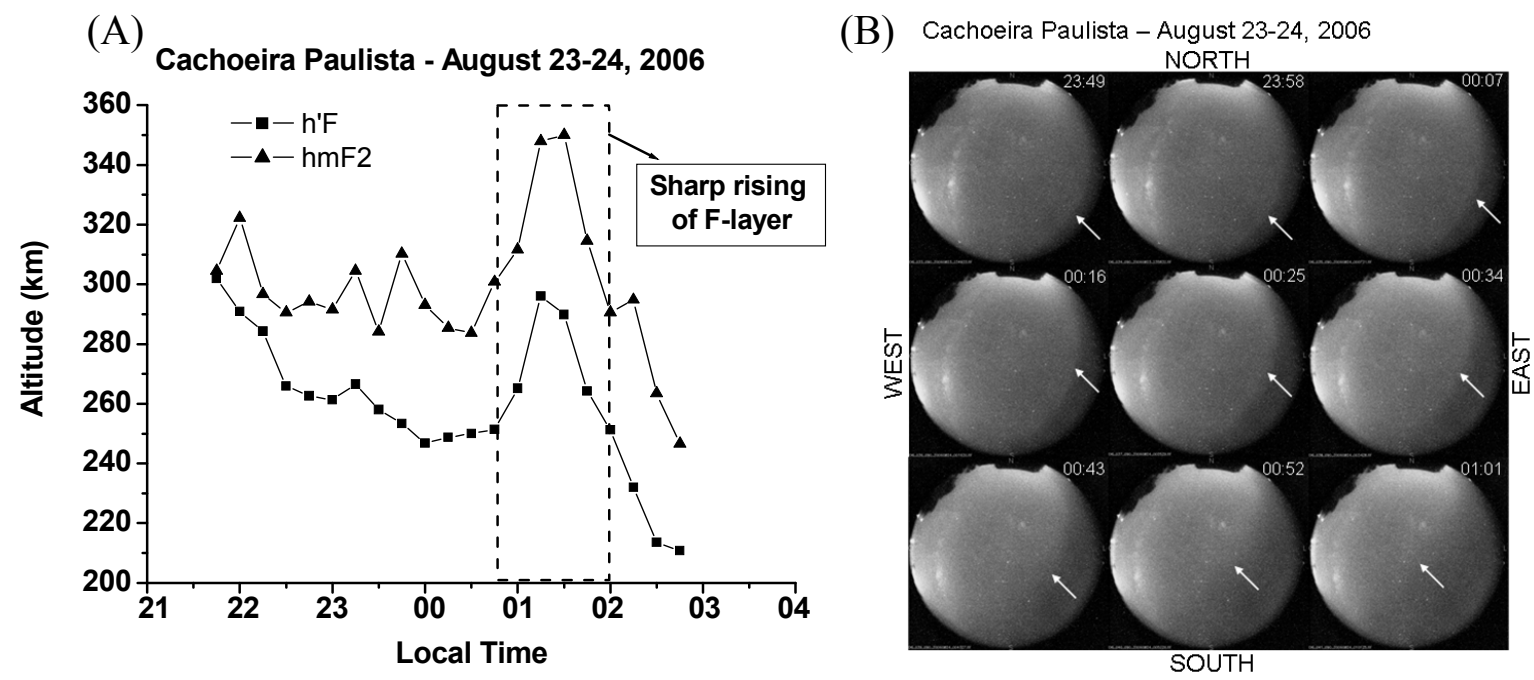

Figure 1 - (a) Temporal variation of the ionospheric parameters h'F and hmF2 for the night of August 23-24, 2006. The dashed rectangle emphasizes the abrupt rise of the F-layer around 01:00 LT. (b) All-sky images of the OI 630 $\mathrm{nm}$ airglow emission for the same night. The dark band structure, indicated by the white arrow, entered from southeast and moved across the field of view towards northwest at an altitude range of 220-300 km.

As mentioned before, the OI $630 \mathrm{~nm}$ emission intensity is strongly dependent on the vertical movements of the ionospheric plasma during the night. Since the OI $630 \mathrm{~nm}$ airglow emission peak is situated at an altitude around $250 \mathrm{~km}$, it is possible to analyze vertical movements of the F-layer plasma through the ionospheric parameters h'F (F-layer bottomside virtual height) and hmF2 (F-layer peak height), provided by ionosonde measurements.

As shown in Figure 1a, the temporal variation of the ionospheric parameters reveals a sharp rising of the ionospheric F-layer. This sharp rising can be caused by upward $\mathbf{E} \times \mathbf{B}$ plasma drifts, driven by a zonal eastward electric field inside the dark band [4].

For all the cases that we have simultaneous data from both all-sky images and ionograms, we have observed the occurrence of spread-F at the same time as the dark bands passes over the zenith of Cachoeira Paulista (example on Figure 2). However, since we do not have simultaneous data for all cases studied, it is not possible to assert the correlation between MSTIDs and spread-F. [5] in a simultaneous analysis of all-sky images and ionograms for two observation sites in the Japanese sector have found only $10 \%$ and $15 \%$ of spread-F for the intervals when the MSTID structures were seen in the airglow images 


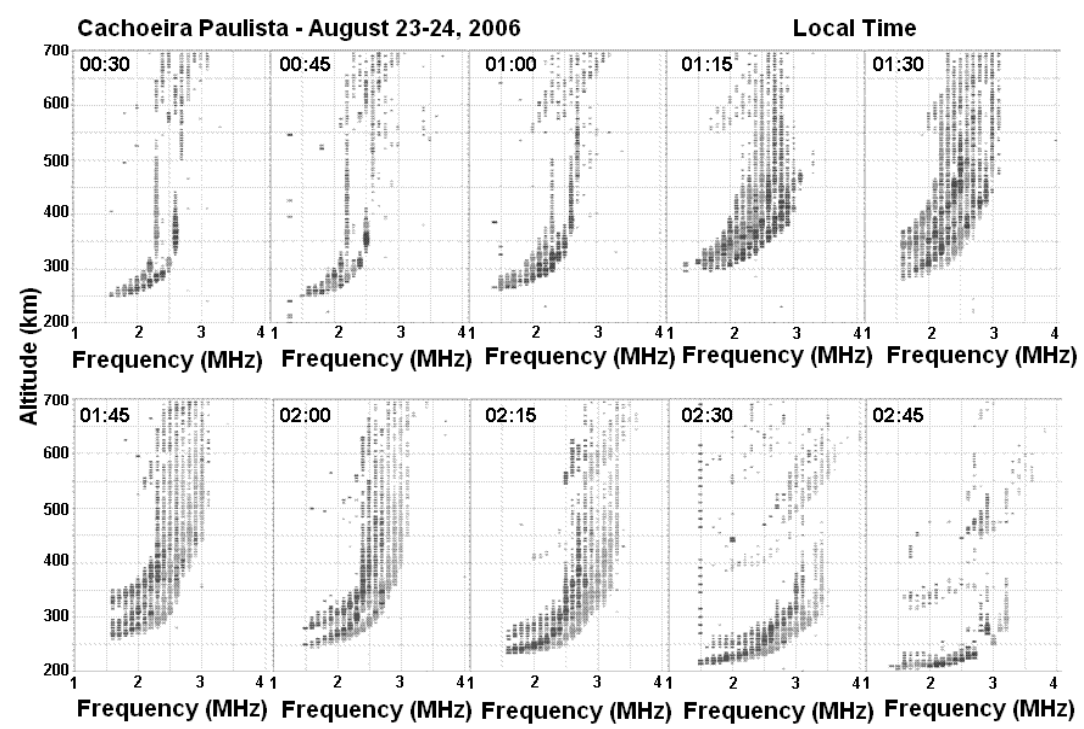

Figure 2 - Ionograms obtained on August 23-24, 2006 with the digisonde installed at Cachoeira Paulista. This sequence shows the occurrence of Spread-F at the same time as the all-sky images registered MSTIDs over the zenith of Cachoeira Paulista, and ionospheric parameters registered abrupt uplifting of both peak height and virtual height of the ionospheric F-layer.

\section{2 investigation of the occurrence of MSTIDs during solar maximum}

Considering the possibility of risings not so sharp of the F-layer during the solar maximum which is not able to disturb the airglow layer, it would not be possible to observe the dark band patterns in the all-sky images. In order to check this possibility, we have made a study based only on ionosonde data, examining the ionospheric parameters h'F and hmF2. Events with features like the occurrence of sharp rises of the F-layer, spread-F in the ionograms, and $\mathrm{Kp}<3$ were considered manifestation of MSTIDs and the computed occurrence rate for the period from March 2000 to February 2001 (solar maximum) is shown on Figure 3.

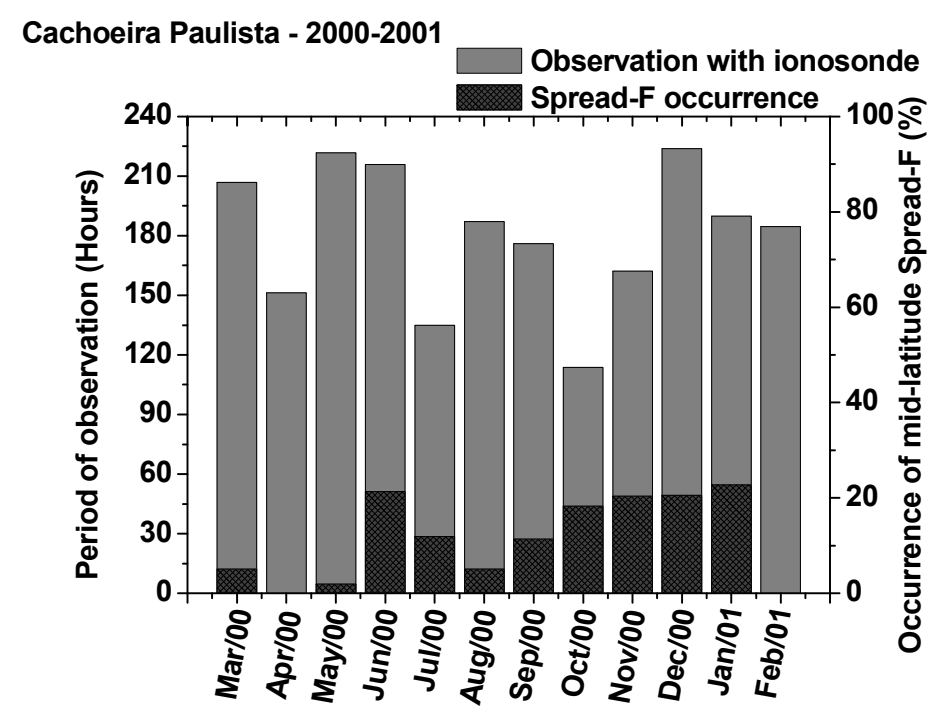

Figure 3 -Statistical analysis of the occurrence of mid-latitude spread-F over Cachoeira Paulista during the period of solar maximum (2000-2001) based on F-region ionograms. 


\section{Discussion}

Ionospheric parameters h'F and hmF2 shows sharp rises of the F-layer during MSTIDs events. These rises are explained as being due to drifts $\mathbf{E}_{\mathbf{p}} \mathbf{x B}$, where $\mathbf{E}_{\mathbf{p}}$ is the polarization electric field inside the MSTID structure. The electrodynamical coupling between the nighttime mid-latitude ionospheric $\mathrm{E}_{\mathrm{s}}$ and F-region have been invoked as an explanation for the large polarization electric fields observed in the F-region and the consequent uplifts of the Flayer which causes spread-F [6]. [7] proposed a mechanism for spread-F development in the mid-latitude ionosphere driven by northward-upward electric fields, which are generated from a meridional polarization field inside an unstable $\mathrm{E}_{\mathrm{s}}$-layer and maps to the F-region via field-lines. [8] derived a dispersion relation that describes the coupling behavior between $\mathrm{E}_{\mathrm{s}}$-layer and Perkins instability. They showed that the coupled system is unstable and the electrodynamics interaction between instabilities acts to increase the growth rate of the global system.

Our analysis regarding the one year ionosonde data set for solar maximum have showed occurrence of MSTIDs during this phase of the solar cycle. Such events show rises not so sharp of F-layer which are not able to diminish the OI $630 \mathrm{~nm}$ emission so that it is not possible to observe the dark band patterns in the all-sky images. Nevertheless, since we do not have comparative data with other instruments, it is not safe to say that all computed cases are manifestation of MSTIDs.

\section{Acknowledgements}

We would like to thank the Ionosphere Group of INPE for kindly providing the ionospheric parameters obtained at Cachoeira Paulista. Also, the authors acknowledge the Conselho Nacional de Desenvolvimento Científico e Tecnológico - CNPq grant 471908/2009-6 and Fundação de Amparo à Pesquisa do Estado de São Paulo - FAPESP grant 2008/50553-8.

\section{References}

1. Pimenta, A. A., M. C. Kelley, Y. Sahai, J. A. Bittencourt, and P. R. Fagundes. "Thermospheric dark band structures observed in all-sky OI $630 \mathrm{~nm}$ emission images over the Brazilian low-latitude sector," Journal of Geophysical Research, 113(A01307), 2008.

2. Pimenta, A. A., D. C. M. Amorim, and C. M. N. Candido. "Thermospheric dark band structures at low latitudes in the Southern Hemisphere under different solar activity conditions: A study using OI $630 \mathrm{~nm}$ emission all-sky images," Geophysical Research Letters, 35(L16103), 2008.

3. Amorim, D.C.M., A.A. Pimenta, J.A. Bittencourt and P.R. Fagundes. "Relevant aspects of MSTIDs observed through OI $630 \mathrm{~nm}$ all-sky imaging over Brazilian low latitudes under different solar activity conditions". Submitted to Journal of Geophysical Research, under review.

4. Kelley, M. C., and J. J. Makela. "Resolution of the discrepancy between experiment and theory of midlatitude Fregion structures," Geophysical Research Letters, 28(13), 2001, pp.2589-2592.

5. Shiokawa, K., C. Ihara, Y. Otsuka, and T. Ogawa. "Statistical study of nighttime medium-scale traveling ionospheric disturbances using midlatitude airglow images," Journal of Geophysical Research, 108 (A1), 2003.

6. Tsunoda, R. T., and R. B. Cosgrove. "Coupled electrodynamics in the nighttime midlatitude ionosphere," Geophysical Research Letters, 28(22), 2001, pp. 4171-4174.

7. Haldoupis, C., M. C. Kelley, G. C. Hussey, and S. Shalimov. "Role of unstable sporadic-E layers in the generation of midlatitude spread F," Journal of Geophysical Research, 108(A12), 2003.

8. Cosgrove, R. B. and R. T. Tsunoda. "Instability of the E-F coupled nighttime midlatitude ionosphere," Journal of Geophysical Research, 109(A04305), 2004. 